\title{
Elastin fragments drive disease progression in a murine model of emphysema
}

\author{
A. McGarry Houghton, ${ }^{1}$ Pablo A. Quintero, ${ }^{1}$ David L. Perkins, ${ }^{2}$ Dale K. Kobayashi, ${ }^{3}$ Diane G. Kelley, ${ }^{3}$ \\ Luiz A. Marconcini, ${ }^{1}$ Robert P. Mecham, ${ }^{4}$ Robert M. Senior, ${ }^{3,4}$ and Steven D. Shapiro ${ }^{1}$
}

1Division of Pulmonary and Critical Care Medicine and 2Division of Nephrology, Brigham and Women's Hospital, Harvard Medical School, Boston, Massachusetts, USA. ${ }^{3}$ Department of Medicine and ${ }^{4}$ Department of Cell Biology, Washington University School of Medicine, St. Louis, Missouri, USA.

\begin{abstract}
Mice lacking macrophage elastase (matrix metalloproteinase-12, or MMP-12) were previously shown to be protected from the development of cigarette smoke-induced emphysema and from the accumulation of lung macrophages normally induced by chronic exposure to cigarette smoke. To determine the basis for macrophage accumulation in experimental emphysema, we now show that bronchoalveolar lavage fluid from WT smoke-exposed animals contained chemotactic activity for monocytes in vitro that was absent in lavage fluid from macrophage elastase-deficient mice. Fractionation of the bronchoalveolar lavage fluid demonstrated the presence of elastin fragments only in the fractions containing chemotactic activity. An mAb against elastin fragments eliminated both the in vitro chemotactic activity and cigarette smoke-induced monocyte recruitment to the lung in vivo. Porcine pancreatic elastase was used to recruit monocytes to the lung and to generate emphysema. Elastin fragment antagonism in this model abrogated both macrophage accumulation and airspace enlargement.
\end{abstract}

\section{Introduction}

Chronic obstructive pulmonary disease (COPD) currently affects over 18 million Americans and is the fourth leading cause of death in the US. The burden of this disease promises to increase throughout the globe as smoking rates continue to climb in most developing countries (1). Emphysema, a major component of COPD, is characterized by an inflammatory cell infiltrate composed of alveolar macrophages, neutrophils, and $\mathrm{CD}^{+}$and $\mathrm{CD} 8^{+}$lymphocytes, and by the presence of proteinases in excess of their inhibitors within the alveolar space, which leads to destruction and permanent enlargement of the peripheral airspaces of the lung (2-7). Macrophages are the predominant inflammatory cell type found in the lung both under normal conditions and in the setting of emphysema (8); however, the mechanisms responsible for the recruitment of peripheral blood monocytes to the lung in the setting of emphysema remain poorly understood.

We have previously shown that mice deficient in macrophage elastase (matrix metalloproteinase-12, or MMP-12) are protected from cigarette smoke-induced emphysema (9). As expected, $M M P-12^{+/+}$mice displayed an increase in alveolar macrophage number in response to cigarette smoke when compared with non-smoke-exposed controls, but surprisingly, $M M P-12^{-/-}$mice did not. This is not simply due to a requirement of MMP-12 for monocytes to properly emigrate from the vasculature to the lung because monocytes do not synthesize MMP-12; only differentiated and activated tissue macrophages produce this proteinase (10). Consistent with this fact, monthly instillations of monocyte chemoattractant protein-1 (MCP-1), concomitant with cigarette smoke exposure, resulted in a significant increase in alveolar macrophages in the MMP-12-/- mice (although they were still pro-

Nonstandard abbreviations used: BAL, bronchoalveolar lavage; BALF, BAL fluid; $\mathrm{CL}$, chord length; COPD, chronic obstructive pulmonary disease; EF, elastin fragment; i.t., intratracheal(ly); MCP, monocyte chemoattractant protein; MMP-12, macrophage elastase (matrix metalloproteinase-12); NE, neutrophil elastase; PPE, porcine pancreatic elastase.

Conflict of interest: The authors have declared that no conflict of interest exists. Citation for this article: J. Clin. Invest. doi:10.1172/JCI25617. tected from the development of emphysema). Thus, $M M P-12^{-}$ monocytes are capable of emigrating to the lung if the appropriate stimulus is present.

In addition to $\mathrm{C}-\mathrm{C}$ chemokine-producing cells, the lung possesses other potential monocyte chemotactic factors that reside within the ECM. Fragments of collagen (11), fibronectin (12), laminin (13), and elastin (14-16) have all been shown to be chemotactic for monocytes in vitro, but data to support a role for these agents in vivo are lacking. In the current study, we show that MMP-12 is required to generate the monocyte chemotactic activity present in a model of cigarette smoke-induced emphysema in the mouse and that elastin fragments (EFs) are required for macrophage accumulation and airspace pathology in this setting.

\section{Results}

Bronchoalveolar lavage fluid from MMP-12+/+ but not MMP-12-/- smokeexposed mice contains monocyte chemotactic activity. Bronchoalveolar lavage fluid (BALF) from WT smoke-exposed mice elicited a 5-fold increase in monocyte chemotaxis in vitro compared with BALF from control mice. In contrast, there was no chemotactic activity in BALF from $M M P-12^{-/-}$smoke-exposed mice above that seen in non-smoke-exposed controls (Figure 1A). Of note, MMP-12 itself was not chemotactic for monocytes in our assay (not shown). Initially, we assayed for C-C chemokines, substances well known for their monocyte chemotactic properties. Real-time PCR was performed on lung RNA from WT mice exposed to smoke for 1, 3, and 6 months, using primers for IL- $1 \beta$, MCP-1, MCP-2, MIP- $1 \alpha$, MIP-1 $\beta$, and RANTES (data not shown). There was no significant increase in RNA expression for any of these chemokines.

Having excluded the common chemokines, we began to search for a novel factor that is generated in $M M P-12^{+/+}$but not $M M P-12^{-/-}$mice in the setting of cigarette smoke exposure. BALF from $M M P-12^{+/+}$ and $M M P-12^{-1-}$ smoke-exposed mice was subjected to gel filtration chromatography. Chemotactic activity was identified only in the BALF from $M M P-12^{+/+}$mice, in fractions corresponding to a molecular mass of approximately $45 \mathrm{kDa}$. Significant chemotactic activity was not present in any fraction of $M M P-12^{-/-}$BALF (Figure 1B). 

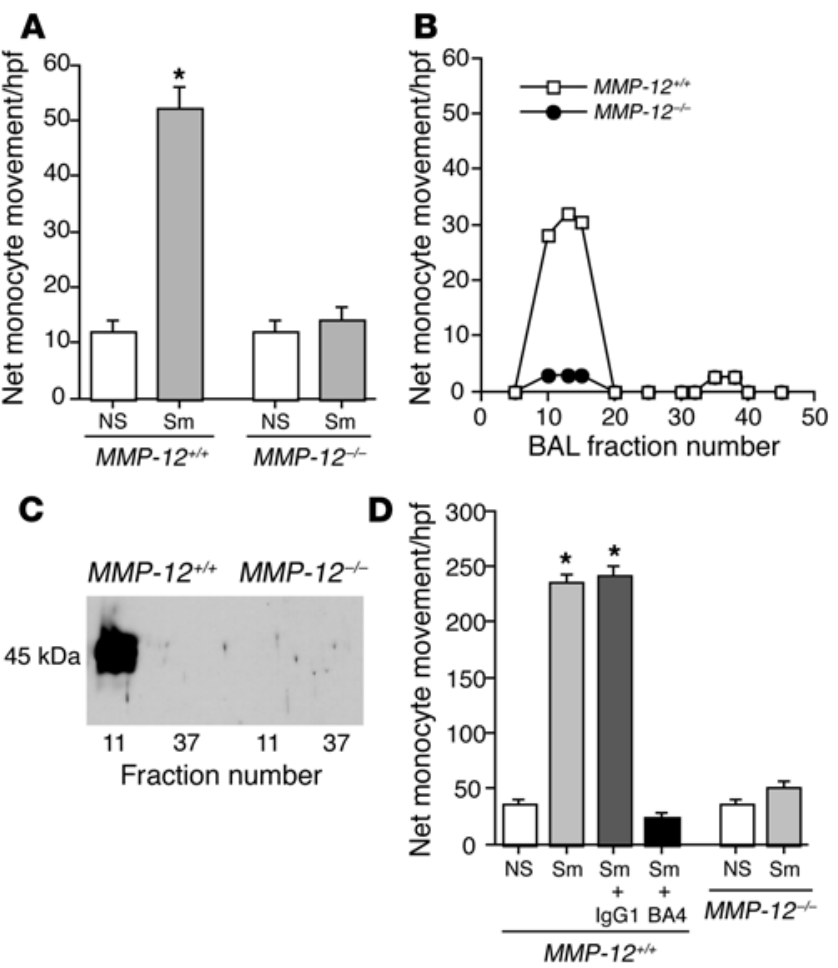

EFs are the chemotactic factor present in BALF. Fractions of the BALF demonstrating chemotactic activity for monocytes in vitro were subjected to Western blot analysis using a panel of antibodies against putative chemokine components of the ECM, including thrombospondin, laminin, collagen, fibronectin, and elastin. Only antibodies directed against elastin were detected in chemotactic fractions (Figure 1C).

Additional in vitro assays of monocyte chemotaxis were performed using lung homogenates from $M M P-12^{+/+}$and $M M P-12^{-/-}$ smoke-exposed animals and non-smoke-exposed controls. Consistent with the results from the experiments using BALF as the chemoattractant, only the lung homogenates from $M M P-12^{+/+}$ mice were chemotactic for monocytes. The lung homogenate demonstrated significantly more potent (5-fold higher) chemotactic activity than the corresponding BALF. To show that EFs are the chemotactic factor present in $M M P-12^{+/+}$smoke-exposed BALF and lung homogenate, we repeated the monocyte chemotaxis assays with the addition of BA4 $\mathrm{mAb}(50 \mathrm{nM})$ to the lower compartments. BA4, an $\mathrm{mAb}$ that was derived from an immunization

\section{Figure 1}

EFs display monocyte chemotactic activity. Human monocytes were isolated by elutriation, and chemotaxis assays were performed in a modified Boyden chamber. (A) BALF from smoke-exposed mice (2 months) was used as the chemoattractant. Data are from a representative experiment performed in triplicate. Net monocyte movement $=$ total monocytes - media control $(=40)$. Bars represent SEM. ${ }^{*} P<0.01$ vs. control. (B) The BAL samples from MMP-12 ${ }^{+/+}$and $M M P-12^{-1-}$ mice were subjected to gel filtration. The fractions were tested for monocyte chemotactic activity (control activity with PBS alone $=0)$. (C) Western blot was performed against gel filtration fractions (nos. 11 and 37 shown here) using a polyclonal anti-elastin antibody. A 45-kDa fragment in MMP-12+/+ active fractions was detected. (D) The assays of monocyte chemotaxis were repeated using lung homogenates from mice exposed to smoke for 2 months as the chemoattractant. BA4 and IgG1 preimmune antibodies were used at a concentration of $50 \mathrm{nM}$. Data are from a representative experiment performed in triplicate. Net monocyte movement per high-powered field $=$ total monocytes - media control $(=46)$. Bars represent SEM. ${ }^{*} P<0.01$ vs. control. hpf, high-powered field; NS, non-smoke exposed; Sm, smoke exposed.

with the $\alpha$-elastin fraction of insoluble elastin, completely eliminated the activity present in BALF and lung homogenate (Figure 1D). However, BA4 had no effect on formyl-Met-Leu-Phe-induced (fMLP-induced) chemotaxis (not shown).

EF inhibition decreases cigarette smoke-induced lung macrophage accumulation in vivo. To illustrate that EFs influence cigarette smokeinduced lung macrophage accumulation in vivo, we used a 1-week cigarette smoke exposure protocol. Acute cigarette smoke exposure experiments are certainly too brief to produce airspace disease or emphysema, but they have been demonstrated to reliably produce an inflammatory cell infiltrate $(17,18)$. Using this protocol, we found that the monocyte recruitment elicited by the chemotactic factors generated by cigarette smoke exposure in vivo can be inhibited $66 \%$ by BA4 $(P=0.0005)$ (Figure 2$)$.

EFs are chemotactic for monocytes in vivo. To further prove that EFs are capable of recruiting peripheral blood monocytes to the lung in vivo, we administered purified EFs (no residual elastase in the digest) intratracheally (i.t.) to WT mice. Intratracheal instillation of EFs resulted in a dose-dependent increase in lung macrophage

\section{Figure 2}

EF antagonism decreases cigarette smoke-induced lung macrophage accumulation in vivo. BAL macrophage counts are shown for mice $(n=5)$ exposed to 4 cigarettes a day for 7 days while receiving daily treatment with BA4, an antibody directed against EFs, or IgG1 control antibody. Negative-control treatments with PBS, IgG1, and BA4 without concomitant cigarette smoke exposure are also shown. Bars represent SEM. ${ }^{\star} P<0.001$ vs. control; ${ }^{\star *} P<0.001$ vs. $\mathrm{Sm}+\operatorname{lgG} 1$.

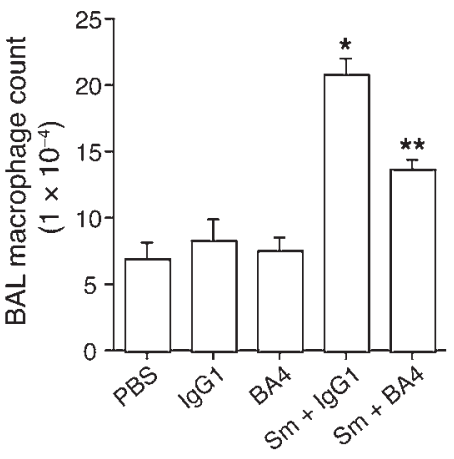


Table 1

Intratracheal instillation of PBS, EFs, and PPE

\begin{tabular}{lccc} 
& $\begin{array}{c}\text { Macrophages/ } \\
\text { mm alveolar wall }\end{array}$ & $\begin{array}{c}\text { BAL macrophages } \\
\left(\mathbf{1 0}^{\mathbf{4}} \mathbf{)}\right.\end{array}$ & $\begin{array}{c}\text { BAL PMNs } \\
\left(\mathbf{1 0 ^ { 4 }}\right)\end{array}$ \\
PBS & $1.74 \pm 0.06$ & $10.24 \pm 1.02$ & 0 \\
PPE $(1 \mathrm{U})$ & $3.99 \pm 0.35^{\mathrm{A}}$ & $18.48 \pm 1.79^{\mathrm{A}}$ & $1.75 \pm 0.26^{\mathrm{A}}$ \\
EF peptides $(10 \mu \mathrm{g})$ & $2.88 \pm 0.13^{\mathrm{A}}$ & $19.85 \pm 0.75^{\mathrm{A}}$ & 0 \\
EF peptides $(100 \mu \mathrm{g})$ & $3.77 \pm 0.22^{\mathrm{A}}$ & $19.63 \pm 2.95^{\mathrm{A}}$ & $0.07 \pm 0.06$ \\
VGVAPG $(5 \mu \mathrm{g})$ & $3.01 \pm 0.13^{\mathrm{A}}$ & - & - \\
EF 45-kDa fragment $(4 \mu \mathrm{g})$ & $4.15 \pm 0.29^{\mathrm{A}}$ & - & - \\
\hline
\end{tabular}

Inflammatory cell counts were tabulated in BAL and on mac-3-stained sections at 72 hours. ${ }^{A} P<0.01$ vs. control. PMN, polymorphonuclear leukocyte.

content both in the BALF and in the lung parenchyma by 72 hours (Table 1). Specifically, EFs, at doses of $10 \mu \mathrm{g}$ and $100 \mu \mathrm{g}$, increased the number of lung macrophages by $65 \%$ and $117 \%$, respectively, compared with PBS-treated controls $(P<0.01)$. Intratracheal administration of VGVAPG, a frequently repeated sequence in tropoelastin that is well known for its chemotactic properties, produced a $73 \%$ increase in lung macrophage content at 72 hours $(P<0.01)$ (Table 1). Furthermore, i.t. administration of the approximately $45-\mathrm{kDa}$ EFs produced a significant increase in lung macrophage content at 72 hours (Table 1). EFs did not recruit neutrophils to the alveolar space above the level seen in controls.

Porcine pancreatic elastase is capable of recruiting monocytes to the lung in vivo. Despite widespread use over the past 30 years, the exact nature of the inflammatory component present in the porcine pancreatic elastase (PPE) model of emphysema has yet to be fully characterized. To prove that PPE generates an inflammatory cell infiltrate, we instilled PPE i.t. and quantified the number of macrophages and neutrophils present in the BALF 1, 3, 7, 14, and 21 days later (Figure 3). A single i.t. dose of PPE $(1 \mathrm{U}=7.4 \mu \mathrm{g})$ produced a significant increase in BALF macrophages (Figure $3 \mathrm{~A}$ ) and neutrophils (Figure 3B) that peaked at 7 and 3 days, respectively. The increase in lung macrophages was also evident in the lung parenchyma on mac-3-stained sections (Figure 3C). Because PPE generates airspace disease, the chord length (CL) was determined so that the cell counts could be corrected for tissue density.

\section{Figure 3}

Effects of PPE and EF antagonism on lung macrophage content in vivo. PPE $(1 \mathrm{U}=7.4 \mu \mathrm{g})$ or PBS control was administered into the lungs of WT mice. BAL was performed before the lungs were inflated and fixed with $10 \%$ buffered formalin at days 1, 3, 7, 14, and 21. (A and B) BAL macrophage (A) and BAL neutrophil (B) counts were tabulated using a hemocytometer and HEMA 3-stained cytospins. (C) Lung macrophage counts are expressed as macrophages per high-powered field adjusted for tissue density on mac-3-stained sections. $n=4$ mice per group. Bars represent SEM. ${ }^{\#} P<0.05$ vs. control. (D) To determine the effect of EF antagonism on monocyte recruitment, PPE-recipient mice received $10 \mu \mathrm{g} \mathrm{BA} 4(n=9), 10 \mu \mathrm{g}$ anti-collagen type I (col) antibody $(n=4), 10 \mu \mathrm{g}$ anti-laminin-5 (lam) antibody $(n=4)$, or $10 \mu \mathrm{g} \operatorname{lgG} 1$ isotype control antibody $(n=8)$ over the course of the 3-day experiment; and either BA4 or IgG1 isotype control over the course of 21 days. The macrophage counts are expressed as above. Bars represent SEM. ${ }^{*} P<0.01$ vs. control; ${ }^{\star \star} P<0.01$ vs. PPE/lgG1. (E-G) Representative mac-3-stained sections are shown for PBS (E), PPE/lgG1 (F), and PPE/BA4 (G) at the 3-day time point. Magnification, $\times 40$.
EF antagonism reduces PPE-induced accumulation of alveolar macrophages in vivo. We used the PPE model of emphysema to study the role of EFs in the recruitment of peripheral blood monocytes to the lung in the setting of experimental emphysema. Intratracheal PPE is well known to induce airspace enlargement, generate EFs, and, as we have shown here, increase lung macrophage content. We administered BA4 i.t. to elastase-recipient mice over either 3 or 21 days, using antibodies against collagen and laminin and an IgG1 isotype antibody as controls. Treatment with BA4 resulted in $62 \%$ and $91 \%$ reduction in lung macrophage content adjusted for alveolar tissue density on mac-3-stained sections at the 3 -day and 21-day time points, respectively, when compared with IgG1 isotype control $(P<0.001)$ (Figure 3, $D-G)$. Collagen and laminin antibodies had no effect on macrophage accumulation in this setting.

EF antagonism abrogates PPE-induced emphysema. Pancreatic elastase causes a direct proteolytic injury to the lung that results in a small increase in airspace size before it is rapidly cleared. The majority of the airspace enlargement present after 21 days $(-80 \%)$ is the result of the chronic inflammatory process initiated by PPE (19). Therefore, modification of this inflammatory process could result in a reduction in the amount of PPE-induced emphysema. To show the
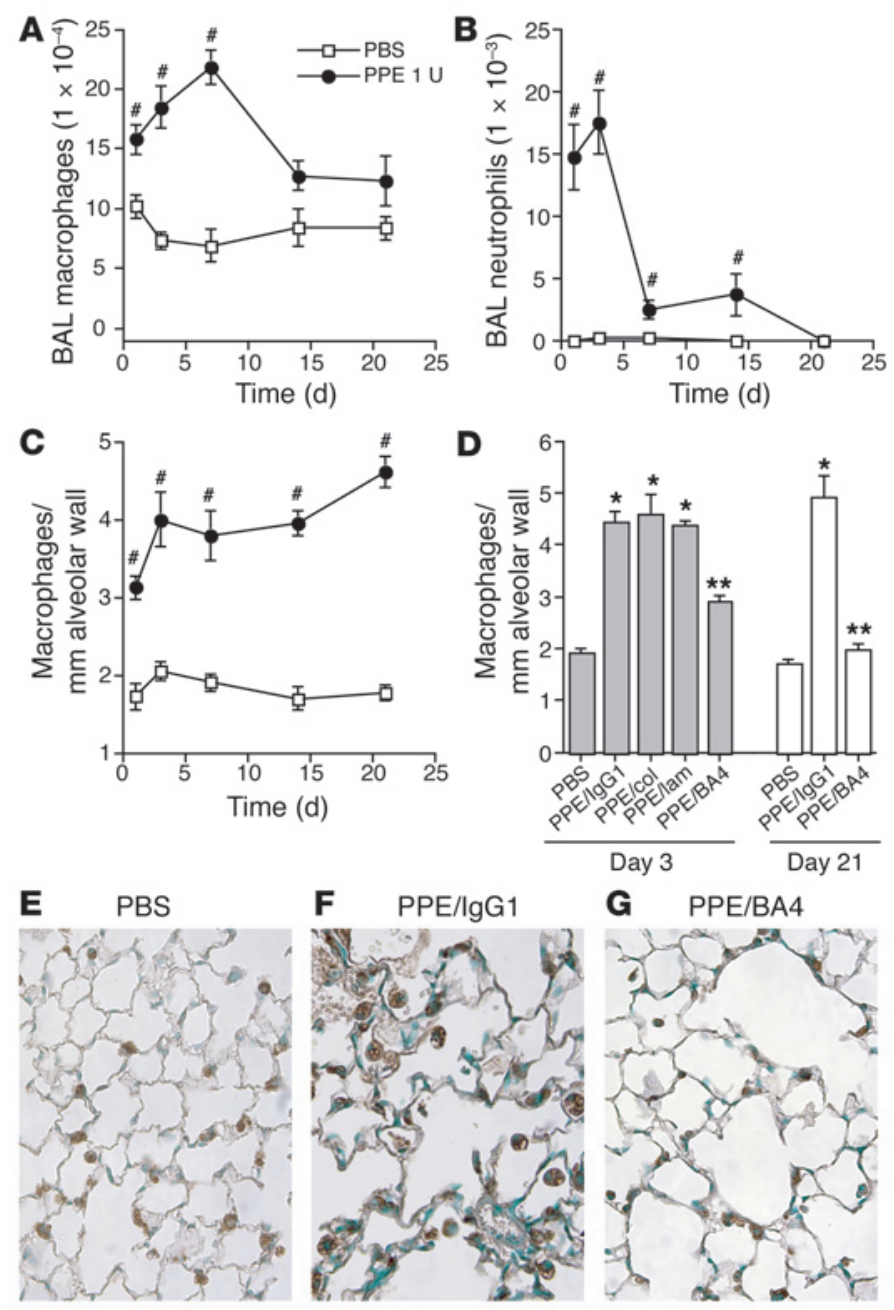

G PPE/BA4

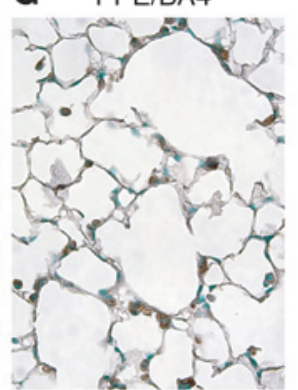



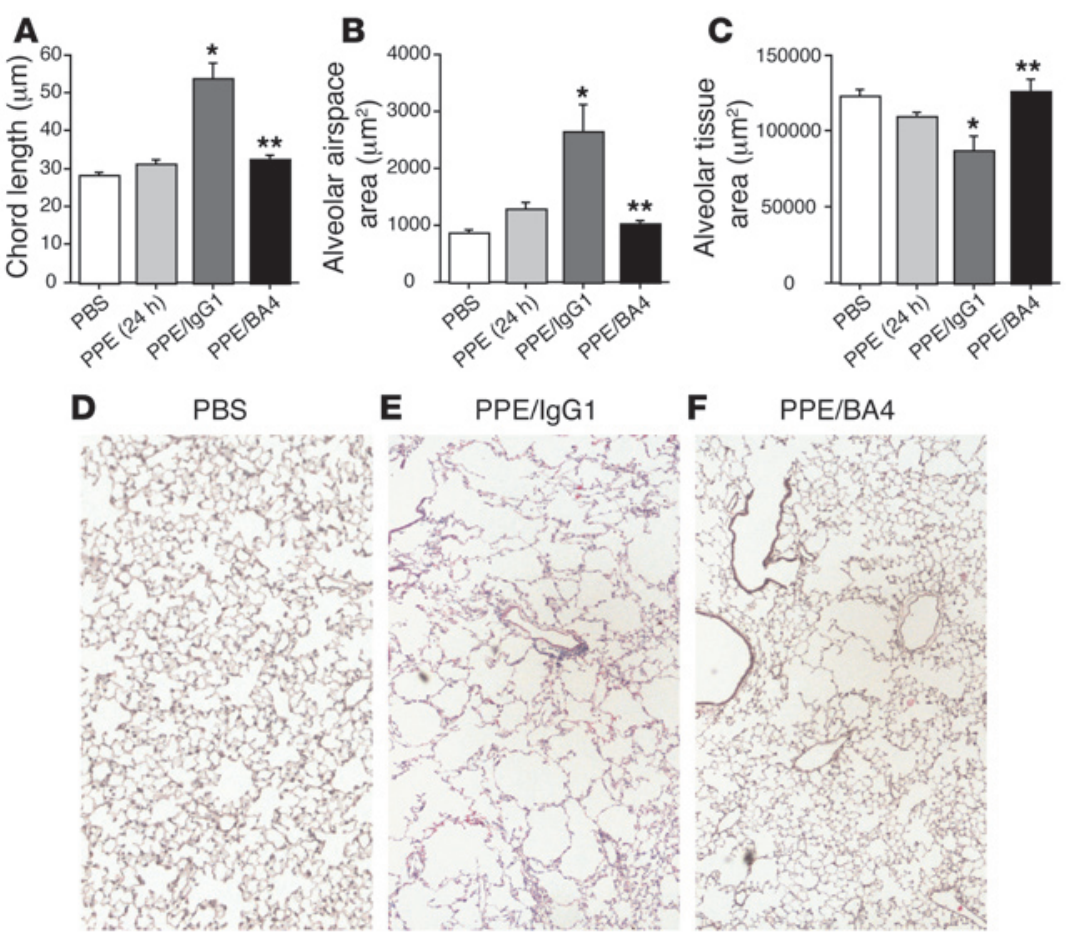

\section{Figure 4}

EF antagonism abrogates PPE-induced emphysema. WT mice received i.t. PPE at time 0 . In addition, the mice received $10 \mu \mathrm{g}$ i.t. doses of $\mathrm{BA} 4$ or IgG1 isotype control on days $1,2,3,5,7,10,13,16$, and 19 ( $n=6$ each group). A separate group of mice received i.t. PBS only at each time point. (A-C) The CLs (A), alveolar airspace areas (B), and alveolar tissue areas (C) are reported for PBS, PPE/lgG1, and PPE/BA4 at the 21-day time point. PPE alone at 24 hours is provided as a reference. Bars represent SEM. ${ }^{\star} P<0.0001$ vs. control; ${ }^{\star \star} P<0.0001$ vs. PPE/lgG1. (D-F) Representative H\&E-stained sections are provided for PBS (D), PPE/IgG1 (E), and PPE/BA4 (F), all at the 21-day time point. Magnification, $\times 10$.

provided (9). Bone marrow transplantation was used to demonstrate that the half-lives of WT and $M M P-12^{-/-}$lung macrophages are equivalent (both are 18 days) and that resident alveolar macrophages do not proliferate, confirming that the accumulation of lung macrophages in our model was due to recruitment of new blood monocytes to the lung and not due to local clonal expansion of resident lung macrophages (data not shown).

The finding that BALF from $M M P-12^{+/+}$, but

dependence of macrophage accumulation and airspace enlargement on the presence of EFs, we repeatedly administered BA4 or isotype control antibody i.t. to PPE-recipient mice and measured the airspace dimensions (CL) and alveolar tissue and airspace areas after 21 days. An additional control group received PBS only at each instillation. BA4 treatment resulted in complete protection from the development of emphysema, with the CL being the same as that in PPE-treated mice after 24 hours, or the part of the injury that is the result of direct proteolysis (Figure 4).

\section{Discussion}

Accumulation of macrophages in the interstitium, septum, and alveolar airspace of the lung is one of the characteristic features of pulmonary emphysema. Over 20 years ago, 2 separate reports by Hunninghake et al. and by members of our group (R.M. Senior and R.P. Mecham) demonstrated that EFs possessed chemotactic properties for monocytes in vitro, but, notably, not for mature alveolar macrophages or neutrophils (14-16). That work fueled speculation that EFs may play a role in the recruitment of monocytes to the lung in emphysema, given that the lung represents a significant source of elastin. Although mature, cross-linked elastic fibers are not chemotactic, cryptic sequences of the protein may be released within the ECM upon proteolysis by elastolytic enzymes, such as MMP-12 or neutrophil elastase (NE). Despite these wellcharacterized chemotactic properties for monocytes in vitro, a role for EFs in vivo in general, and in the pathogenesis of emphysema in particular, has remained purely speculative. Moreover, with the discovery of the large $\mathrm{C}-\mathrm{C}$ chemokine family, the role of matrix fragments in mediating chemotaxis seemed minimal, at best.

We entertained a number of potential mechanisms that could explain the discrepancy that we observed in lung macrophage content between WT and MMP-12-/- smoke-exposed mice. We have previously shown that MMP-12-/- peripheral blood monocytes are capable of emigrating to the lung when an appropriate stimulus is not from $M M P-12^{-/-}$, mice contained monocyte chemotactic activity led us to search initially for known, and later for novel, chemotactic factors that were generated only in the $M M P-12^{+/+}$ mice. We were able to detect only fragments of elastin, a known substrate for MMP-12 (20), in chemotactic fractions of bronchoalveolar lavage (BAL). Inhibition of monocyte chemotaxis using BA4 confirmed the dependence of this chemotactic activity on EFs. EF inhibition is relevant in vivo as well, given that BA4 administration concomitant with cigarette smoke exposure significantly reduced the accumulation of lung macrophages.

To demonstrate that EF-driven macrophage accumulation can inhibit the development of emphysema, we turned to the PPE model of emphysema. The PPE model, used to reliably generate experimental emphysema for decades, has been criticized in the past for rapidly producing airspace disease via direct proteolytic

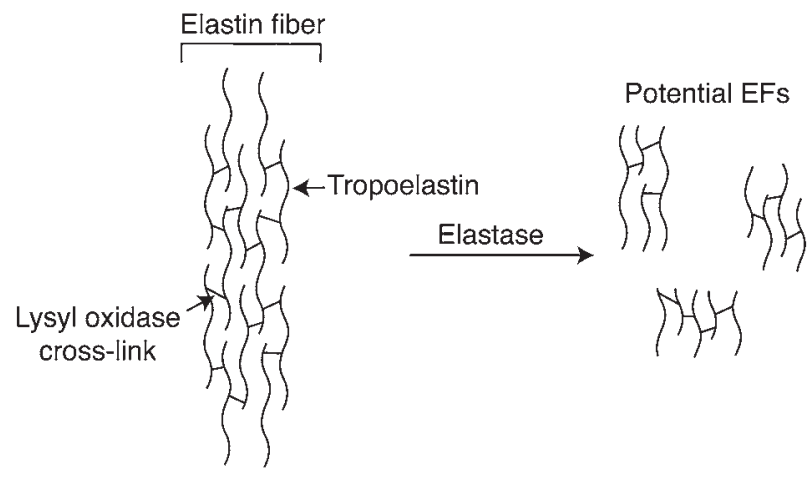

Figure 5

Depiction of an elastin fiber. The schematic depicts an elastin fiber that consists of multiple tropoelastin monomers cross-linked together by lysyl oxidase. Potential degradation products and their cross-linked nature are shown. 
destruction of the alveolar wall, and thus having little in common with the chronic, low-grade inflammation observed in emphysema. However, recent data suggest that the amount of emphysema generated by direct proteolytic tissue destruction by PPE is minimal, compared with the lesion that develops over the ensuing 3-4 weeks related to endogenous inflammation and destruction. Modification of this inflammatory cascade has been demonstrated to significantly reduce the resultant airspace disease (19), suggesting that PPE generates a chronic inflammatory condition that more closely resembles that of human emphysema than was previously thought. We used the PPE model of emphysema here to show that EF inhibition over a 3-week time course significantly decreases the accumulation of lung macrophages and eliminates the development of airspace pathology, with the amount of airspace disease present in the BA4 group being similar to that present 24 hours after the administration of PPE, or the part of the injury that is the direct result of PPE-mediated proteolysis. EF antagonism reduced PPE-induced lung macrophage accumulation by $65 \%$ and $90 \%$ at 3 and 21 days, respectively. Prolonged treatment with BA4, therefore, achieves a more complete reduction in macrophage number, but there certainly exists an alternative mechanism that would account for the remainder. We have not been unable to detect significant increases in the C-C chemokines, so there is likely an alternate, as-yet unrecognized chemotactic factor.

EFs exert their chemotactic influence on monocytes via interaction with the elastin-binding protein (EBP) (21). Whereas the hexapeptide VGVAPG, a commonly repeated sequence in human elastin, is the most well-recognized ligand for this receptor, numerous other EFs are capable of binding to the EBP. In addition to VGVAPG, all elastin-derived peptides that follow the motif GXXPG or XGXPG (where $X$ is a hydrophobic amino acid) display chemotaxis for monocytes in vitro $(22,23)$. This is noteworthy because the murine tropoelastin sequence does not contain the VGVAPG sequence; however, it does contain significant quantities of both GXXPG and XGXPG. In fact, these commonly repeated sequences represent $21 \%$ of the entire mouse tropoelastin sequence. Also of note, epitope recognition studies have demonstrated that BA4 also binds to all peptides that follow the GXXPG and XGXPG motifs (24), thus explaining why it is an effective inhibitor of the chemotactic EFs generated in the mouse. Although the monocyte chemotactic properties of EFs reside within these hydrophobic penta- and hexapeptide repeats, the EFs generated in vivo tend to be much larger. In the current study, we identified EFs in the BALF from WT smoke-exposed mice in gel filtration fractions of approximately $45 \mathrm{kDa}$ (the broad peak of activity ranged from 35 to $50 \mathrm{kDa}$ ). Prior studies have demonstrated a wide range in the size of EFs that possess monocyte chemotactic activity, from 10 to $50 \mathrm{kDa}$, that seems to depend on the type of elastin digested and the enzyme used to digest it (14-16).

Mature elastin is a complex polymer of tropoelastin monomers that have been irreversibly cross-linked by the enzyme lysyl oxidase. As a result, the mature protein is highly insoluble, and fragments generated from the polymer by proteolysis (Figure 5) are heterogeneous in size and composition (the broad band detected here is likely the result of several distinct fragments between 35 and $50 \mathrm{kDa}$, not 1 unique EF). Most contain multiple chain fragments joined through 1 or more cross-links, making their characterization by conventional proteomic approaches difficult if not impossible (25). Given the extensive prior work showing that the chemotactic properties of EFs reside within the commonly repeat- ed penta- and hexapeptides, and that BA4 effectively inhibits them both in vitro and in vivo, it is likely that the larger peptides we identified contain the chemotactic sequences cross-linked with other regions of the protein. Furthermore, additional analysis of substrate specificities for MMP-12, within elastin, may provide insight into the unique structural features of EFs generated in vivo.

Based on our previous findings and our current study of MMP-12-/- mice, we hypothesize that alveolar macrophages become activated upon chronic exposure to cigarette smoke; that these macrophages release MMP-12 that degrades ECM components including elastin; and that the degradation of elastin results in the generation of EFs chemotactic for monocytes. The early influx of neutrophils and NE caused by cigarette smoke exposure (17, 18) also contributes to the generation of EFs and provides a possible explanation as to why $\mathrm{NE}^{-/-}$mice are partially protected from both the accumulation of macrophages and the development of airspace enlargement upon exposure to cigarette smoke (26). The interplay between macrophages and neutrophils in this process is unlikely to be limited to the generation of EFs. We have previously shown that NE is capable of degrading the tissue inhibitors of metalloproteinases (TIMPs), the major inhibitors of MMP-12. Furthermore, MMP-12 degrades $\alpha 1$-antitrypsin, whose major function is to inhibit NE. Thus it appears that these proteinases are able to indirectly augment each other's function by degrading one another's inhibitors. However, it is not surprising that the lack of MMP-12 would prevent the generation of cigarette smoke-induced EFs in our model, given that the recruitment of neutrophils (and $\mathrm{NE}$ ) by cigarette smoke in mice is modest, when compared with macrophage accumulation. Therefore, given the sparse representation of neutrophils in the cigarette smoke-induced emphysema in our mice that are housed in a sterile barrier facility, the greater relative importance of the macrophage and its degradative enzymes is not surprising. Whereas PPE administration elicits both macrophage and neutrophil inflammation (Figure 3), the neutrophils are cleared rapidly in this setting, apparently leaving the majority of alveolar tissue destruction to macrophages, which maintain their upregulation, presumably via continued production of EFs. It is also possible that EFs induce MMP biosynthesis (27). This EF chemotactic signal ultimately leads to enhanced lung tissue destruction by placing more macrophages, and thus more MMP-12, within close proximity of the airspace. In the absence of repair, peripheral airspace enlargement is the result (28).

Although the findings here are limited to a model of emphysema, the concept that degradation products of elastin can drive a macrophage-predominant, chronic inflammatory disease process is potentially applicable to all diseases that occur in elastin-rich organs, including coronary artery disease, peripheral vascular disease, and aortic aneurysm (29). Additionally, EF antagonism represents a potential therapeutic strategy for COPD, a disease for which, to date, there are no available disease-modifying agents.

\section{Methods}

Materials. All reagents were obtained from Sigma-Aldrich unless otherwise noted. BA4 is a mouse anti-bovine IgG1-subclass mAb that was derived from an immunization with the $\alpha$-elastin fraction of insoluble elastin. It has subsequently been shown to recognize the VGVAPG hexapeptide found in human elastin, among others (24).

Mice. MMP-12-deficient mice and WT littermates were used for the experiments involving cigarette smoke exposure. The $M M P-12^{-/-}$mice were generated in our laboratory as previously described (10). Briefly, intron 1 and 
exon 2 were disrupted by the insertion of the phosphoglycerolkinase promoter and neomycin resistance genes (PGK-neo). The construct was electroporated into RW4 ES cells, and correctly targeted clones were microinjected into C57BL/6J blastocysts. Mice homozygous for the MMP-12 mutation were backcrossed 10 generations into the C57BL/6J background. Age- and sex-matched WT littermates were used as controls. All other experiments were carried out in C57BL/6J mice purchased from Jackson Laboratory. The Harvard Standing Committee for Animal Research at Harvard University School of Public Health approved all animal experiments.

Cigarette smoke exposure. $M M P-12^{+/+}$and $M M P-12^{-/-}$mice $(n=10)$ were exposed to cigarette smoke from 2 unfiltered cigarettes (University of Kentucky) 6 days a week for 1, 2, 3, or 6 months (depending on the experiment) using a smoking apparatus as previously described (9). The animals tolerated the treatment without evidence of toxicity (carboxyhemoglobin levels $10 \%$ ). We additionally used an acute cigarette smoke exposure protocol in which the mice were subjected to the smoke from 4 cigarettes a day for 7 days.

$B A L F$ analysis. At the conclusion of a given experiment, mice were sacrificed before performance of BAL via a 22-gauge i.v. catheter inserted into the trachea. The lungs were lavaged with $1 \mathrm{ml}$ DMEM 3 times to obtain the BALF. The BALF was then centrifuged at maximum speed for 3 minutes, and the supernatant was retained for further analysis. BALF obtained in this way was subjected to gel filtration using an HPLC machine (Waters Corp.) with a HiLoad Superdex 200 column (Amersham Biosciences). Each BAL sample was loaded in a volume of $1 \mathrm{ml}$ into a bed volume of $320 \mathrm{ml}$. Fractions were collected in 1-ml aliquots using a flow rate of $1 \mathrm{ml} / \mathrm{min}$ in a buffer composed of $50 \mathrm{mM}$ Tris ( $\mathrm{pH} 7.5$ ), $10 \mathrm{mM} \mathrm{CaCl}_{2}$, and $150 \mathrm{mM} \mathrm{NaCl}$. Using this technique, the BALF was separated into 56 fractions based on molecular weight. Each of the fractions was assayed for chemotactic properties as described below. Representative fractions (nos. 11 and 37) of the BALF (100x concentration) were then subjected to Western blot analysis using enhanced chemiluminescence. Polyclonal anti-elastin (rabbit antimouse) antibody at a 1:1,000 dilution was used for Western blot analysis. BA4 $\mathrm{mAb}$ (described above) at a 1:1,000 dilution was used to confirm these results. In addition, the BALF was analyzed for cellular content. The BALF was centrifuged at $1,900 \mathrm{~g}$ for 3 minutes, the rbcs were lysed, and the BAL was resuspended in $1.0 \mathrm{ml}$ normal saline. Macrophage, neutrophil, and total cell counts were determined using a hemocytometer and cytospins stained with HEMA 3 (Biochemical Sciences Inc.).

Mouse lung elastin was digested using human leukocyte elastase (both Elastin Products Co.) and subjected to gel filtration in a protocol similar to that described above. The fraction corresponding to a molecular weight of approximately $45 \mathrm{kDa}$ was administered i.t. (see below) to demonstrate its monocyte chemotactic properties in vivo. Tris buffer control was not significantly different from PBS control (not shown).

Monocyte chemotaxis. Assays of monocyte chemotaxis were performed on human peripheral blood monocytes using either BALF or lung homogenate from WT and MMP-12-/- smoke-exposed and non-smoke-exposed control mice as the chemoattractants in separate experiments. Human peripheral blood monocytes were obtained by phlebotomy and purified using an elutriation centrifugation protocol. Monocytes were placed in the top wells of a microchemotaxis chamber at a concentration of $1.0 \times 10^{5}$ cells per well in DMEM. Depending on the assay, the lower compartments contained either (a) $M M P-12^{+/+}$versus $M M P-12^{-/-}$unfractionated BALF from smokeexposed and non-smoke-exposed control mice normalized to total protein content of $2 \mathrm{mg} / \mathrm{ml}$; (b) $M M P-12^{+/+}$versus $M M P-12^{-/-}$BALF fraction (nos. 1-56) from smoke-exposed and non-smoke-exposed controls normalized to total protein content of $8 \mu \mathrm{g} / \mathrm{ml}$; or (c) $M M P-12^{+/+}$versus $M M P-12^{-/-}$lung homogenate from smoke-exposed and non-smoke-exposed control mice. The chemotaxis assays using unfractionated BALF and lung homogenates were repeated with the addition of $50 \mathrm{nM}$ BA4 to the lower compartment at the initiation of the experiment. Results are from a representative experiment performed in triplicate.

Real-time PCR. WT mice $(n=6)$ were exposed to cigarette smoke as described above for 1,3 , or 6 months. Age- and sex-matched WT mice were used as non-smoke-exposed controls. The lungs were removed and frozen at $-80^{\circ} \mathrm{C}$. The lungs were homogenized in $1 \mathrm{ml}$ cold TRIzol prior to RNA isolation according to the manufacturer's instructions. After treatment with DNase I (Ambion Inc.) to remove contaminating DNA, the samples were reversetranscribed using RT-PCR beads with Poly(dT) oligonucleotides according to the manufacturer's instructions (Amersham Biosciences). Real-time PCR analysis was performed using the GeneAmp 5700 Sequence Detection System (Applied Biosystems). The comparative CT method was employed using $\mathrm{GAPDH}$ as an endogenous reference housekeeping gene. Serial dilutions of a control sample of cDNA were used as the standard curve for each reaction. SYBR Green buffer (Applied Biosystems) was used as the fluorophore. All experiments were performed in triplicate. The primer sequences were as follows: IL-1 $\beta$, forward primer, $5^{\prime}$-CCTCTGGTGTGTGACGTTC-3'; reverse primer, 5'-TCTCGGAGCCTGTAGTGCAGT-3'; MCP-1, forward primer, 5' TTCTGGGCCTGCTGTTCAC-3'; reverse primer, 5'-GAGCCAACACGTGGATGCT-3'; MCP-2, forward primer, 5'-TCTCCCCTAATCTTCTCCAGG$3^{\prime}$; reverse primer, 5'-CAGGCACCATCTGCTTGTAAC-3'; MIP-1 $\alpha$, forward primer, 5'-CTGCCTGCTGCTTCTCCTACA-3'; reverse primer, 5'-CAACGATGAATTGGCGTGG-3'; MIP-1 $\beta$, forward primer, $5^{\prime}$-TCTCTCCTCTT-

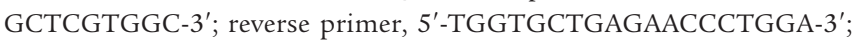
RANTES, forward primer, $5^{\prime}$-GCAAGTGCTCCAATCTTGCA-3'; reverse primer, 5'-CTTGGCGGTTCCTTCGAGT-3'. Results are expressed as the fold change from non-smoke-exposed controls.

Intratracheal administration of EFs, PPE, and BA4. WT mice were anesthetized with an i.p. injection of $2.5 \%$ Avertin. After the mice were appropriately sedated, they were intubated via direct visualization of the larynx using a 22-gauge i.v. catheter in which the needle point had been filed down to a blunt end and was used as a stylet. One unit $(7.4 \mu \mathrm{g})$ PPE; $10 \mu \mathrm{g}$ or 100 $\mu \mathrm{g}$ purified digested bovine ligament elastin (i.e., EFs); $5 \mu \mathrm{g}$ VGVAPG (all from Elastin Products Co.); the approximately $45-\mathrm{kDa}$ EF (generated as described above); or PBS or Tris buffer control ( $n=4-6$ for each group) was administered i.t. in a total volume of $40 \mu \mathrm{l}$.

To demonstrate the dependence of monocyte recruitment to the lung on EFs in the PPE model of emphysema, we administered BA4 to inhibit the chemotactic properties of EFs. On day 1, WT mice were anesthetized with $2.5 \%$ Avertin and received a dose of $10 \mu \mathrm{g}$ BA4 $(n=9), 10 \mu \mathrm{g} \mathrm{IgG1}$ isotype control antibody $(n=8), 10 \mu \mathrm{g}$ anti-collagen type I antibody (Calbiochem) $(n=4)$, or $10 \mu \mathrm{g}$ anti-laminin-5 antibody (R\&D Systems) $(n=4)$ i.t. as described above. Two hours later, all of the animals received a single i.t. dose of PPE (1.0 U). On days 2 and 3, the mice again received i.t. doses of BA4 or isotype control. The animals were sacrificed after 72 hours.

To evaluate the effects of EF inhibition on the progression of airspace disease, we coadministered BA4 or $\operatorname{IgG} 1$ isotype control antibody i.t. to PPE-recipient mice. In this experiment, mice received a single dose of i.t. PPE $(1.0 \mathrm{U})$ at time 0 and then received BA4 or control antibody on days 1 , $2,3,5,7,10,13,16$, and 19 . The animals were sacrificed on the 21 st day.

In a separate experiment, mice received either i.t. BA4 or IgG1 isotype control concurrent with an acute cigarette smoke exposure protocol (described above).

Lung tissue preparation. At the conclusion of each experiment, the lungs were inflated to allow for further analysis. The animals were sacrificed at the appropriate time point, the RV was perfused with normal saline to remove blood, and the lungs were inflated at $25 \mathrm{~cm} \mathrm{H}_{2} \mathrm{O}$ with $10 \%$ buffered formalin for 10 minutes via an i.t. catheter. The lungs were then removed and fixed for 24 hours before embedding in paraffin. Serial mid-sagittal sections were obtained for morphological and histological analysis. 
In addition, lung tissue homogenates were prepared from mice exposed to smoke for 2 months, by homogenization of the lungs in $5 \mathrm{ml}$ of $50 \mathrm{mM}$ Tris, $150 \mathrm{mM} \mathrm{NaCl}$, and $10 \mathrm{mM} \mathrm{CaCl}_{2}$ buffer.

Morphometry. Mid-sagittal sections stained with a modified Gill's stain were used to calculate the CL as previously described (9). Ten randomly selected ×200 fields per slide were photographed using MetaMorph (Molecular Devices). The images were analyzed using Scion Image (Scion Corp.). Airway and vascular structures were eliminated from the analysis. The images were manually thresholded. The CLs were determined in both a horizontal and a vertical plane, allowing for the calculation of alveolar airspace areas.

Immunohistochemistry. Alveolar, interstitial, and septal macrophages were quantified with mac-3 immunostaining (rat anti-mouse antibody at 1:1,000 dilution; BD Biosciences - Pharmingen) on mid-sagittal sections using the avidin-biotin HRP technique in which 3,3'-diaminobenzidine was the chromogenic substrate. Results are represented as the average count from 5 different high-powered fields per slide that have been corrected for millimeters of alveolar wall using the morphometric data (as described above).

1. Peto, R., Chen, Z.M., and Boreham, J. 1999. Tobacco: the growing epidemic. Nat. Med. 5:15-17.

2. Ofulue, A.F., and Ko, M. 1999. Effects of depletion of neutrophils or macrophages on development of cigarette smoke-induced emphysema. Am. J. Physiol. 21:L97-L105.

3. Merchant, R.K., Schwartz, D.A., Helmers, R.A., Dayton, C.S., and Hunninghake, G.W. 1992. Bronchoalveolar lavage cellularity. Am. Rev. Respir. Dis. 146:448-453.

4. Dhami, R., et al. 2000. Acute cigarette smokeinduced connective tissue breakdown is mediated by neutrophils and prevented by $\alpha 1$-antitrypsin. Am. J. Respir. Cell Mol. Biol. 22:244-252.

5. Saetta, M., et al. 1998. CD8+ T-lymphocytes in peripheral airways of smokers with chronic obstructive pulmonary disease. Am. J. Respir. Crit. Care Med. 157:822-826.

6. Hogg, J.C., et al. 2004. The nature of small-airway obstruction in chronic obstructive pulmonary disease. N. Engl. J. Med. 350:2645-2653.

7. Retamales, I., et al. 2001. Amplification of inflammation in emphysema and its association with latent adenoviral infection. Am. J. Respir. Crit. Care Med. 164:469-473.

8. Rodriguez, R.J., White, R.R., Senior, R.M., and Levine, E.A. 1977. Elastase release from human alveolar macrophages: comparison between smokers and non-smokers. Science. 198:313-314.

9. Hautamaki, R.D., Kobayashi, D.K., Senior, R.M., and Shapiro, S.D. 1997. Requirement for macrophage elastase for cigarette smoke-induced emphysema in mice. Science. 277:2002-2004.

10. Shipley, J.M., Wesselschmidt, R.L., Kobayashi, D.K., Ley, T.J., and Shapiro, S.D. 1996. Metalloelastase is required for macrophage-mediated proteolysis and matrix invasion in mice. Proc. Natl. Acad. Sci. U. S. A.

Statistics. Data are expressed as the mean value \pm SEM unless otherwise indicated. Statistical significance was determined using the Student's $t$ test (2-tailed distribution with a 2 -sample equal variance). $P$ values less than 0.05 were considered statistically significant.

\section{Acknowledgments}

This work was supported in part by grants from the NIH and the National Heart, Lung, and Blood Institute (29594 to S.D. Shapiro and R.M. Senior) and by the Edith L. Wolff Charitable Trust (R.M. Senior).

Received for publication May 12, 2005, and accepted in revised form December 6, 2005.

Address correspondence to: A. McGarry Houghton, Brigham and Women's Hospital, 75 Francis Street, Boston, Massachusetts 02115, USA. Phone: (617) 278-0765; Fax: (617) 232-4623; E-mail: mhoughton@rics.bwh.harvard.edu.

93:3942-3946.

11. Postlethwaite, A.E., and Kang, A.H. 1976. Collagen and collagen peptide-induced chemotaxis of human blood monocytes. J. Exp. Med. 143:1299-1307.

12. Clark, R.A.F., Wikner, N.E., Doherty, D.E., and Norris, D.A. 1988. Cryptic chemotactic activity of fibronectin for human monocytes resides in the $120-\mathrm{kDa}$ fibroblastic cell-binding fragment. J. Biol. Chem. 263:12115-12123.

13. Adair-Kirk, T.L., et al. 2003. A site on laminin $\alpha 5$, AQARSAASKVKVSMKF, induces inflammatory cell production of matrix metalloproteinase- 9 and chemotaxis. J. Immunol. 171:398-406.

14. Senior, R.M., Griffin, G.L., and Mecham, R.P. 1980. Chemotactic activity of elastin-derived peptides. J. Clin. Invest. 66:859-862.

15. Senior, R.M., et al. 1984. Val-Gly-Val-Ala-Pro-Gly, a repeating peptide in elastin, is chemotactic for fibroblasts and monocytes. J. Cell Biol. 99:870-874.

16. Hunninghake, G.W., et al. 1981. Elastin fragments attract macrophage precursors to diseased sites in pulmonary emphysema. Science. 212:925-927.

17. Churg, A., et al. 2002. Acute cigarette smokeinduced connective tissue breakdown requires both neutrophils and macrophage metalloelastase in mice. Am. J. Respir. Cell Mol. Biol. 27:368-374.

18. Churg, A., Dai, J., Tai, H., Xie, C., and Wright, J.L. 2002. Tumor necrosis factor- $\alpha$ is central to acute cigarette smoke-induced inflammation and connective tissue breakdown. Am. J. Respir. Crit. Care Med. 166:849-854.

19. Lucey, E.C., Keane, J., Kuang, P., Snider, G.L., and Goldstein, R.H. 2002. Severity of elastase-induced emphysema is decreased in tumor necrosis factor$\alpha$ and interleukin- $1 \beta$ receptor-deficient mice. $L a b$. Invest. 82:79-85.

20. Mecham, R.P., et al. 1997. Elastin degrada- tion by matrix metalloproteinases. J. Biol. Chem. 272:18071-18076.

21. Privitera, S., Prody, C.A., Callahan, J.W., and Hinek, A. 1998 . The $67-\mathrm{kDa}$ enzymatically inactive alternatively spliced variant of beta-galactosidase is identical to the elastin/laminin-binding protein. J. Biol. Chem. 273:6319-6326.

22. Bisaccia, F., et al. 1994. Migration of monocytes in the presence of elastolytic fragments of elastin and in synthetic derivates. Int. J. Pept. Protein Res. 44:332-341.

23. Castiglione Morelli, M.A., et al. 1997. Structureactivity relationships for some elastin-derived peptide chemoattractants. J. Pept. Res. 49:492-499.

24. Grosso, L.E., and Scott, M. 1993. Peptide sequences selected by BA4, a tropoelastin-specific monoclonal antibody, are ligands for the 67-kilodalton bovine elastin receptor. Biochemistry. 32:13369-13374.

25. Brown-Augsburger, P., Tisdale, C., Broekelmann, T., Sloan, C., and Mecham, R.P. 1995. Identification of an elastin cross-linking domain that joins three peptide chains. J. Biol. Chem. 270:17778-17783.

26. Shapiro, S.D., et al. 2003. Neutrophil elastase contributes to cigarette smoke-induced emphysema in mice. Am. J. Pathol. 163:2329-2335.

27. Brassart, B., et al. 2001. Conformational dependence of collagenase (matrix metalloproteinase-1) up-regulation by elastin peptides in cultured fibroblasts. J. Biol. Chem. 276:5222-5227.

28. Liu, X., et al. 2004. Elastic fiber homeostasis requires lysyl oxidase-like 1 protein. Nat. Genet. 36:178-182.

29. Hance, K.A., Tataria, M., Ziporin, S.J., Lee, J.K., and Thompson, R.W. 2002. Monocyte chemotactic activity in human abdominal aortic aneurysms: role of elastin degradation peptides and the $67-\mathrm{kD}$ cell surface elastin receptor. J. Vasc. Surg. 35:254-261. 